\title{
NUCLEAR DATA SENSITIVITY/UNCERTAINTY PRE-ANALYSIS OF FNG WCLL FUSION BENCHMARK
}

\author{
Ivan A. Kodeli ${ }^{*}$, Maurizio Angelone ${ }^{2}$, Davide Flamini ${ }^{2}$ \\ ${ }^{1}$ Jožef Stefan Institute, Ljubljana, Slovenia \\ ${ }^{2}$ ENEA, Frascati (Rome), Italy \\ ivan.kodeli@ijs.si, maurizio.angelone@enea.it, davide.flamini@enea.it
}

\begin{abstract}
To assure tritium self-sufficiency in future fusion reactors such as DEMO the accuracy of TRP calculations has to be demonstrated within the design uncertainties. A new neutronics experiment representing a mock-up of the Water Cooled Lithium Lead (WCLL) Test Blanket Module (TBM) is under preparation at the Frascati neutron generator (FNG) with the objective to provide an experimental validation of accuracy of nuclear data and neutron transport codes for the tritium production rate (TPR) calculations. The mock-up will consist of LiPb bricks, EUROFER plates and Perspex substituting water. The mock-up will be irradiated by $14 \mathrm{MeV}$ neutrons at the FNG facility, and the TPR and detector reaction rates will be measured using $\mathrm{Li}_{2} \mathrm{CO}_{3}$ pellets and activation foils placed at different positions up to about $55 \mathrm{~cm}$ inside the mock-up. Computational pre-analyses for the design of the WCLL neutronics experiment using the SUSD3D sensitivity/uncertainty $(\mathrm{S} / \mathrm{U})$ code system is described and compared with the results of some similar FNG experiments performed in the past, in particular the FNG HCPB Tritium Breeder Module Mock-up (2005) and FNG-HCLL Tritium Breeder Module Mock-up (2009). The objective of the pre-analysis is to provide the calculated nuclear responses including the uncertainties due to the uncertainties in nuclear data and thus contributes to the optimisation of the design of the experimental set-up.
\end{abstract}

KEYWORDS: nuclear data, sensitivity /uncertainty analysis, benchmarks

\section{INTRODUCTION}

Since $20+$ years a series of neutronics benchmark experiments have been performed at the Frascati neutron generator (FNG) of ENEA Frascati in the scope of the European fusion programme [1-7]. Nuclear data sensitivity and uncertainty $(\mathrm{S} / \mathrm{U})$ analyses were integral part of the benchmark experiment analysis since the very beginning of this experimental programme, and were used both for the pre- and post-analyses of the experiments for the optimization of the benchmark set-up and the interpretation of the measured results, respectively. Examples of the successful use of deterministic and Monte Carlo sensitivity methods for benchmark analysis include FNG-ITER Blanket Bulk Shield (1995), FNG-ITER Streaming (1997/98), FNG Silicon Carbide (2001), FNG Tungsten (2002), FNG HCPB Tritium Breeder Module Mock-up (2005), FNG-HCLL Tritium Breeder Module Mock-up (2009) and FNG-Copper (2015). All these benchmarks are already included in the SINBAD database, or in the process of being included (FNG Cu and FNG HCLL).

Present address: Culham Centre for Fusion Energy, Abingdon, UK 
Tritium self-sufficiency which will be needed for DEMO reactor is still to be demonstrated, taking into account both the losses of the tritium inventory as well as the uncertainties in the tritium production. Two concepts of the Test Blanket Module (TBM) considered within the EU fusion technology programme for tritium production (breeding) in the fusion power demonstration reactor (DEMO) were already tested at FNG. The Helium-Cooled Pebble Bed (HCPB) Breeder Blanket mock-up benchmark experiment was performed in 2005 and consists of a metallic beryllium set-up with two double layers of breeder material ( $\mathrm{Li}_{2} \mathrm{CO}_{3}$ powder). The reaction rate measurements include the $\mathrm{Li}_{2} \mathrm{CO}_{3}$ pellets for the tritium breeding monitoring and activation foils, inserted at several axial and lateral locations in the block. The experiment and the results of the analysis using the Monte Carlo and deterministic transport, sensitivity and uncertainty code system were presented in [1-3].

The tritium breeding-module helium-cooled lithium-lead benchmark experiment (TBM HCLL) was performed in 2009 [3-7]. The HCLL mock-up consisted of a block of $45 \mathrm{~cm}$ x $51.66 \mathrm{~cm}$ side-view and 34.6 $\mathrm{cm}$ long, placed $5.3 \mathrm{~cm}$ in front of the $14 \mathrm{MeV}$ FNG neutron source. The block was composed of 11 alternating layers of LiPb bricks (3.6 cm high) and EUROFER-97 (Reduced Activation Ferritic/Martensitic Steel) plates $(0.915 \mathrm{~cm}$ thick). Two additional thin layers and a back reflector of Polyethylene have been introduced. Several independent measurements of the TPR were performed by ENEA, FZK/TUD and JAEA. Other independent techniques have also been used, such as Thermo Luminescence Detectors (TLDs), through distinct measurements of the absorbed dose due both to the energy released in the $(n, t)$ reaction and in the decay of tritium, and diamond detectors covered with ${ }^{6} \mathrm{LiF}$. The neutron flux attenuation in the PbLi has been measured using the activation foil technique.

A new neutronics experiment representing a mock-up of the Water Cooled Lithium Lead (WCLL) TBM [8] is under preparation at the FNG facility with the objective to experimentally validate the capability of the neutronics codes and nuclear data to predict the tritium production rate (TPR) and other nuclear responses within the design uncertainties. The mock-up will consist of LiPb bricks, EUROFER plates and Perspex substituting water (polyethylene-type material, $\mathrm{C}_{5} \mathrm{O}_{2} \mathrm{H}_{8}$ ). As in the above mentioned cases, the mock-up will be irradiated by $14 \mathrm{MeV}$ neutrons at the FNG facility, and the TPR and detector reaction rates will be measured using $\mathrm{Li}_{2} \mathrm{CO}_{3}$ pellets (containing both natural and ${ }^{6} \mathrm{Li}$-enriched lithium) and activation foils placed at various positions inside the mock-up. Fast and the thermal neutron flux will be measured using the $\mathrm{Al}$, $\mathrm{Ni}, \mathrm{Nb}, \mathrm{In}, \mathrm{Au}$ activation foils. The TPR will be measured both with the natural $\left(7.5 \%{ }^{6} \mathrm{Li}\right)$ and $95 \%$ enriched $\mathrm{Li}_{2} \mathrm{CO}_{3}$ pellets. Sets of pellets are planned to be introduced at 7 axial positions starting from $\sim 8$ $\mathrm{cm}$ to $\sim 50 \mathrm{~cm}$ in the mock-up.

Computational pre-analyses for the design of the WCLL neutronics experiment using the SUSD3D S/U code system was performed to assess the uncertainty in tritium production rate (TPR) due to the uncertainty in the relevant nuclear data and compared with the results of some similar FNG experiments performed in the past, in particular the FNG-HCLL Tritium Breeder Module Mock-up (2009). The objective of the preanalysis is to provide calculated nuclear responses including uncertainties and thus contributes to the optimization of the design of the experimental set-up. The reference neutron transport calculations were performed with the MCNP6 code accelerated using weight window parameters.

\section{SENSITIVITY-UNCERTAINTY ANALYSIS}

Like in the previous FNG benchmarks, the preparation of the FNG-WCLL experiment started with the preanalyses which have been performed both by deterministic and Monte Carlo [8] codes. The pre-analysis includes the simulation of the planned benchmark experiment using a detailed model of the assembly and of the experimental set up in order to determine the sensitivity of the reaction rates to be measured in the mock-up to the underlying cross sections, and the associated uncertainties. The sensitivity and uncertainty analysis of the experiment reveals the most relevant elements/isotopes and nuclear reactions that most 
contribute to the determination of the TPR, which shall permit to optimize the geometry, the detector positions and the choice of activation reactions, and in the post-analysis phase to interpret the results of the measurements and the calculations and to conclude on the quality of available nuclear data, such as the recent JEFF evaluations.

The nuclear data sensitivity and uncertainty analysis was performed by means of the SUSD3D $[9,10]$ code system which was standardly used for most previous FNG benchmark pre- and post-analyses. SUSD3D is a deterministic cross section sensitivity code based on the Generalized perturbation theory. For previous FNG benchmarks the intercomparison of the SUSD3D deterministic calculations with the Monte Carlo approach available in the MCSEN5 [11] code showed excellent agreement.

The SUSD3D [9] code requires as input quantities the direct and adjoint angular moment fluxes calculated by the Discrete ordinates codes (such as DORT-TORT [12], PARTISN, DENOVO), as well as the cross sections and covariance matrices for the relevant materials and reactions. As in the previous analysis of the FNG benchmarks the reference $\mathrm{S} / \mathrm{U}$ calculations were based on the direct and adjoint neutron fluxes calculated using the DOORS discrete ordinates package which includes, among others, the DORT (2D) and TORT (3D) transport solvers. Due to the complex geometry the three-dimensional rectangular (XYZ) geometry model was simulated using the TORT code. At this stage of the analysis the dosimeters were not explicitly included in the model. The GRTUNCL3D [13] code was used to mitigate ray effects which are likely to occur due to the $14 \mathrm{MeV}$ neutron source place in the air in front of the mock-up. Consistent with the past experience the $\mathrm{S}_{16}$ and $\mathrm{P}_{5}$ approximations were adopted for the transport calculations. Nuclear cross-section data taken from the JEFF-3.3 [14] evaluation were prepared in the 175 VITAMIN-J energy group structure and processed by the TRANSX code [15]. The response functions and the corresponding covariances were taken from IRDF-2002 [16] and IRDFF [17]. JEFF 3.3 and SCALE-6 [18] covariance matrix data were used for the cross-sections.

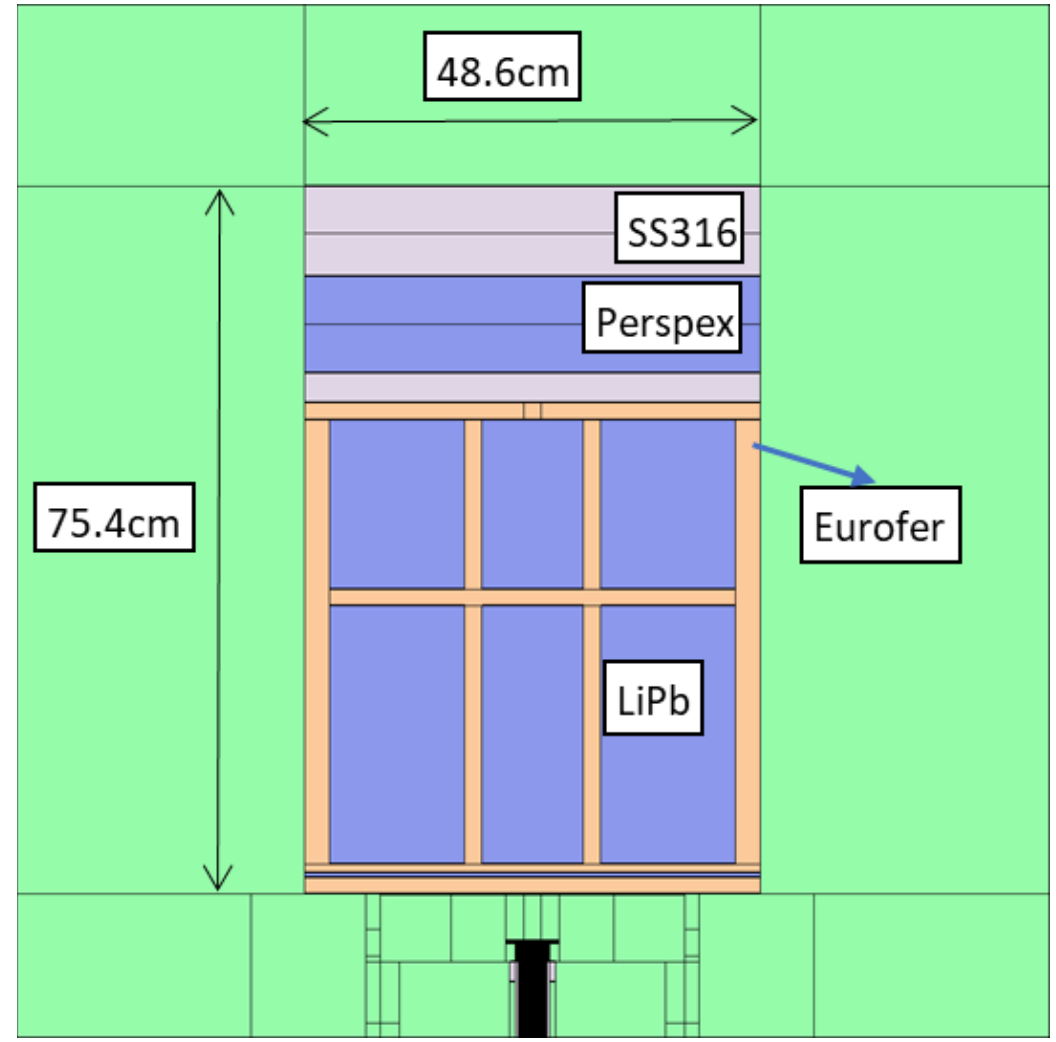

Figure 1: View of the FNG WCLL geometrical model. 
Preliminary calculations were also performed using the new S/U scheme based on the ADVANTG and DENOVO codes [19]. At this stage the calculations were based on the 33 energy group calculations (JEFF 3.3) with limited number of groups at the fast energy range. These analyses will be continued with higher number of groups, but the preliminary results are encouraging and show good consistency between the approaches.

The reference transport calculations were done using the detailed MCNP [20] computational model [8]. In addition, a similar model is also under preparation for the SERPENT code, demonstrating consistent (preliminary) results. The agreement between the reaction rates calculated with the TORT code and the MCNP results is reasonably good, mostly within 10 to $20 \%$, with higher differences for the ${ }^{197} \mathrm{Au}(\mathrm{n}, \gamma)$ reaction rates showing a systematic underestimation of around $50 \%$ on the average. The reason for these discrepancies are under investigation, with probable reasons in the thermal cross section and/or selfshielding treatment.

\subsection{Cross-section sensitivity analysis}

Cumulative reaction rate integrals, their sensitivity to the cross sections, as well as the uncertainties were estimated for selected detector working positions in the experimental block, in particular for deep penetrations where the highest uncertainties are expected. The sensitivity of the ${ }^{6} \operatorname{Li}(n, t),{ }^{7} \operatorname{Li}\left(n, n^{\prime} t\right),{ }^{115} \operatorname{In}\left(n, n^{\prime}\right)$ and ${ }^{27} \mathrm{Al}(\mathrm{n}, \alpha)$ reactions rates with respect to the underlying cross sections was analysed. ${ }^{115} \operatorname{In}\left(\mathrm{n}, \mathrm{n}^{\prime}\right)$ and ${ }^{27} \mathrm{Al}(\mathrm{n}, \alpha)$ were chosen because of their different energy spectra coverage (intermediate and fast energy range, respectively) which was expected to lead to different cross section sensitivities and uncertainties. To evaluate the impact of considerable differences between the spectra at the front and back positions the sensitivity of the TPR in Li was also calculated for the front position. Few examples of sensitivity profiles are shown on Figure 2.

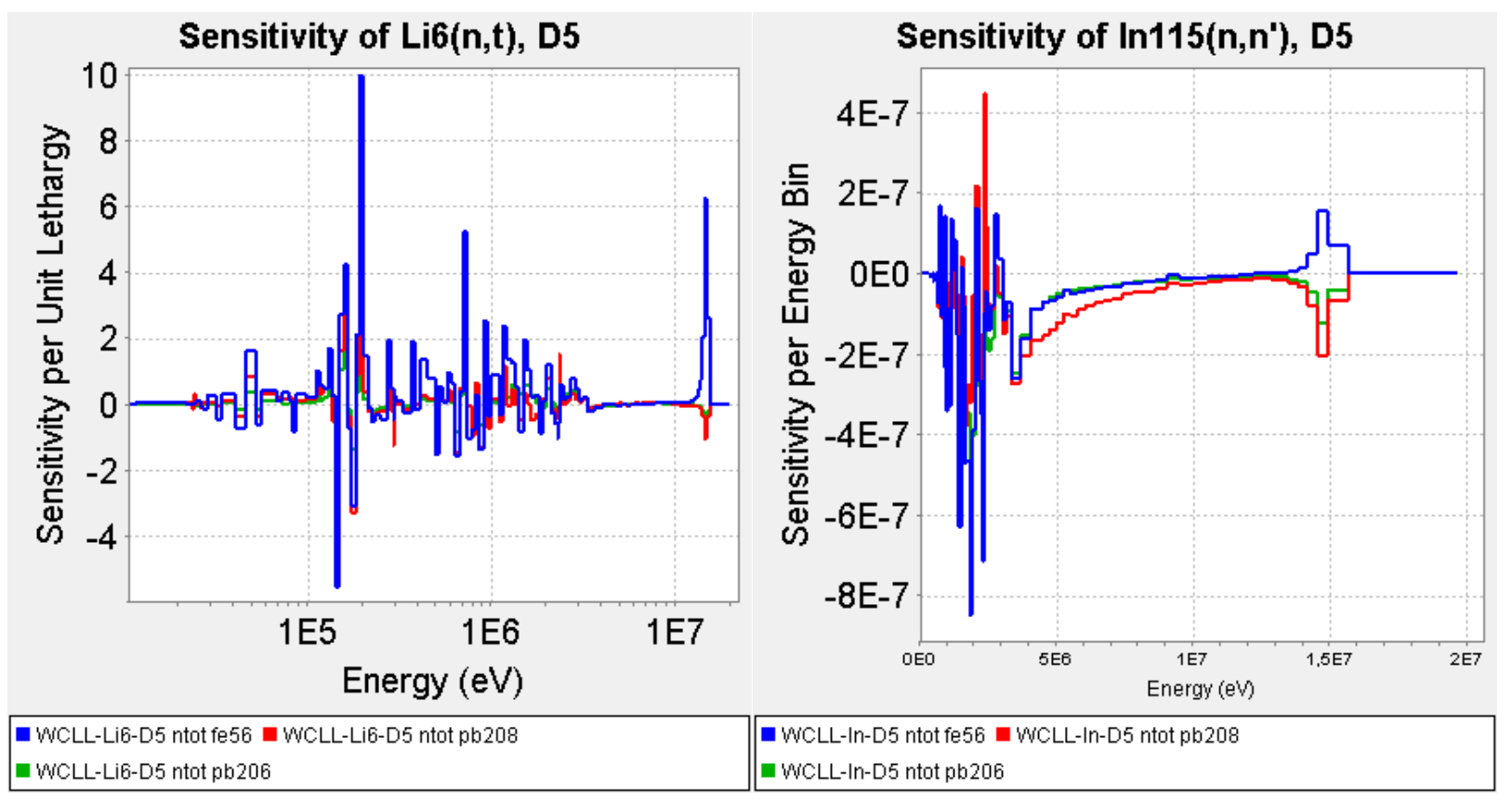

Figure 2: Examples of sensitivities of the ${ }^{6} \mathrm{Li}(\mathrm{n}, \mathrm{t})$ and ${ }^{115} \operatorname{In}(\mathrm{n}, \mathrm{n})$ ') reaction rates at the position D5 (46 cm in the block) with respect to the $\mathrm{Pb}$ and Fe total cross sections. 
The above reactions rates were found to be most sensitive to the following partial cross sections:

- $\quad{ }^{6} \mathrm{Li}(\mathrm{n}, \mathrm{t})$ at $\mathrm{D} 2$ and $\mathrm{D} 5$ : elastic scattering on ${ }^{56} \mathrm{Fe},{ }^{208,206,207} \mathrm{~Pb},{ }^{1} \mathrm{H}$ (front positions)

- ${ }^{7} \mathrm{Li}\left(\mathrm{n}, \mathrm{n}\right.$ 't) at D2 and D5: in particular $(\mathrm{n}, 2 \mathrm{n})$ and inelastic scattering on ${ }^{208,206,207} \mathrm{~Pb},{ }^{56} \mathrm{Fe}$

- ${ }^{115} \mathrm{In}\left(\mathrm{n}, \mathrm{n}^{\prime}\right)$ at D5: inelastic scattering on ${ }^{208,206,207} \mathrm{~Pb},{ }^{56} \mathrm{Fe}$

- ${ }^{27} \mathrm{Al}(\mathrm{n}, \alpha)$ at $\mathrm{D} 5:(\mathrm{n}, 2 \mathrm{n})$ reaction on ${ }^{208,206,207} \mathrm{~Pb},{ }^{56} \mathrm{Fe}$.

\subsection{Cross section uncertainty analysis}

To calculate the uncertainties in the calculated reaction rates the above sensitivities were folded with the cross section covariance matrices taken from the recent JEFF-3.3 nuclear data evaluation. NJOY [21] code was used to process the covariances in the 175 energy groups used in the neutron transport and sensitivity calculations. Older covariances from the ZZ-SCALE6.0/COVA-44G library [18], extracted from the SCALE-6 package, were also used for comparison, mainly because they were used in the analysis of some previous benchmark experiments. However, the data were primarily developed for thermal reactor studies and as such they are not suitable for fusion applications. The covariances are given in 44 energy groups, and were converted into 175 group structure using the ANGELO 2.3 code [24].

The uncertainties for several calculated neutron reaction rates with respect to major reaction cross-sections are presented in Tables I and II. From Table I we identify the nuclear cross sections mostly contributing to the uncertainties in the calculations as follows:

- ${ }^{6} \mathrm{Li}(\mathrm{n}, \mathrm{t})$ at D2 and D5: elastic on ${ }^{56} \mathrm{Fe}$ and ${ }^{208} \mathrm{~Pb}$ elastic, inelastic scattering on ${ }^{208,206,207} \mathrm{~Pb},{ }^{56} \mathrm{Fe}$

- $\quad{ }^{7} \mathrm{Li}\left(\mathrm{n}, \mathrm{n}\right.$ 't) at D2 and D5: (n,2n) reaction on ${ }^{208,206,207} \mathrm{~Pb}$, inelastic scattering on ${ }^{208,206,207} \mathrm{~Pb},{ }^{7} \mathrm{Li}$,

- ${ }^{115} \mathrm{In}\left(\mathrm{n}, \mathrm{n}\right.$ ') at D5: inelastic scattering on ${ }^{208,206,207} \mathrm{~Pb},(\mathrm{n}, 2 \mathrm{n})$ reaction on ${ }^{208,206,207} \mathrm{~Pb}$, inelastic on ${ }^{56} \mathrm{Fe}$

- $\quad{ }^{27} \mathrm{Al}(\mathrm{n}, \alpha)$ at D5: $(\mathrm{n}, 2 \mathrm{n})$ reaction on ${ }^{208,206,207} \mathrm{~Pb}$, inelastic scattering on ${ }^{208,206,207} \mathrm{~Pb},{ }^{56} \mathrm{Fe}$

Table I. Main contributors to uncertainty in the calculated detector reaction rates due to uncertainties in neutron cross sections. Ten largest contributors are listed for each detector reaction (SUSD3D preliminary results based on the TORT neutron fluxes $(\mathrm{D} 2=7.7 \mathrm{~cm}, \mathrm{D} 5=46 \mathrm{~cm}$ ).

\begin{tabular}{|c|c|c|c|c|c|c|c|}
\hline \multirow[b]{2}{*}{ Isotope } & \multirow[b]{2}{*}{ Reaction } & \multicolumn{6}{|c|}{ Uncertainty (\%) } \\
\hline & & $\begin{array}{c}{ }^{6} \operatorname{Li}(\mathrm{n}, \mathrm{t}) \\
\mathrm{D} 2\end{array}$ & $\begin{array}{c}{ }^{6} \operatorname{Li}(\mathrm{n}, \mathrm{t}) \\
\mathrm{D} 5\end{array}$ & $\begin{array}{c}{ }^{7} \mathrm{Li}\left(\mathrm{n}, \mathrm{n}{ }^{\prime} \mathrm{t}\right) \\
\text { D2 }\end{array}$ & $\begin{array}{c}{ }^{7} \operatorname{Li}(n, n ’ t) \\
\text { D5 }\end{array}$ & $\begin{array}{c}{ }^{115} \operatorname{In}\left(\mathrm{n}, \mathrm{n}^{\prime}\right) \\
\text { D5 }\end{array}$ & $\begin{array}{c}{ }^{27} \mathrm{Al}(\mathrm{n}, \mathrm{a}) \\
\mathrm{D} 5\end{array}$ \\
\hline${ }^{208} \mathrm{~Pb}$ & inelast. & 0.9 & 2.0 & 1.2 & 6.9 & 5.9 & 5.9 \\
\hline & elast. & 2.2 & 2.8 & & & 0.3 & 0.3 \\
\hline & $(n, 2 n)$ & 0.5 & 1.4 & 5.2 & 22.0 & 4.2 & 17.3 \\
\hline${ }^{207} \mathrm{~Pb}$ & inelast. & 0.9 & 2.2 & 0.6 & 3.1 & 4.5 & 3.7 \\
\hline & $(n, 2 n)$ & & 0.7 & 2.6 & 10.8 & 2.1 & 8.4 \\
\hline${ }^{206} \mathrm{~Pb}$ & inelast. & 2.0 & 4.7 & 0.6 & 3.2 & 8.4 & 3.7 \\
\hline & elast. & 0.4 & & & & 0.3 & \\
\hline & $(n, 2 n)$ & & & 2.6 & 10.6 & 1.9 & 8.6 \\
\hline${ }^{56} \mathrm{Fe}$ & inelast. & 0.8 & 1.8 & & 1.3 & 1.8 & 0.8 \\
\hline & elast. & 2.7 & 7.8 & & & 0.4 & \\
\hline & $(n, 2 n)$ & 0.4 & 0.7 & 0.8 & 0.9 & & 0.7 \\
\hline & $(n, \gamma)$ & & 1.7 & & & & \\
\hline${ }^{1} \mathrm{H}$ & elast. & 0.6 & & & & & \\
\hline${ }^{7} \mathrm{Li}$ & total & & & 1.5 & 1.5 & & 0.2 \\
\hline
\end{tabular}


Note that natural and $95 \%$ enriched Li pellets will be used for the TPR measurements. In spite of the high uncertainties in TPR from ${ }^{7} \mathrm{Li}$ due to nuclear data of $\mathrm{Fe}$ and $\mathrm{Pb}$ isotopes the contribution of ${ }^{7} \mathrm{Li}$ to the total TPR (and therefore to the uncertainty) was found to be nevertheless relatively small as compared to ${ }^{6} \mathrm{Li}$, both for $95 \%{ }^{6} \mathrm{Li}$ enriched as well as for natural samples. The uncertainty in the total TPR is therefore dominated by the uncertainty in ${ }^{6} \mathrm{Li}(\mathrm{n}, \mathrm{t})$ and limited to between around 5 to $7 \%$ both for the natural and enriched pellets.

For comparison, the uncertainties based on ENDF/B-VIII covariance data are added in Table II. Note however that ENDF/B-VIII evaluation at present does not include covariance information for some isotopes (e.g. ${ }^{56} \mathrm{Fe}$ in particular) therefore the corresponding uncertainties may be slightly underestimated since restricted to ${ }^{206,207,208} \mathrm{~Pb}$ covariances only.

Table III summarizes the uncertainties calculated for the FNG HCLL benchmark [6,7] using SCALE-6 covariance data. The measurement uncertainties were of the order of around $5 \%$ for the reaction rates and TPR. As reported in $[3,4]$ the observed C/E values, generally within $5 \%$ for the reaction rates and $\sim 10 \%$ for the TPR, were in agreement with the above computational and measurement uncertainties.

Comparing with the uncertainties given in Table II for the planned FNG WCLL benchmark we can furthermore observe considerably higher predicted computational uncertainties for the new benchmark, suggesting that FNG WCLL is likely to contribute importantly to the validation and improvement of nuclear data.

Table II. Total uncertainty in the calculated reaction rates for FNG WCLL benchmark based on JEFF3.3 and SCALE-6.0 covariance matrix evaluations (SUSD3D preliminary results based on the TORT 3D neutron fluxes, D2 $=7.7 \mathrm{~cm}, \mathrm{D} 5=46 \mathrm{~cm}$ ).

\begin{tabular}{|c|c|c|c|c|c|c|}
\hline \multirow{2}{*}{ WCLL } & \multicolumn{7}{|c|}{ Uncertainty $(\%)$} \\
\cline { 2 - 7 } Covariance data & ${ }^{115} \operatorname{In}\left(\mathrm{n}, \mathrm{n}^{\prime}\right)$ & ${ }^{27} \mathrm{Al}(\mathrm{n}, \alpha)$ & ${ }^{6} \operatorname{Li}(\mathrm{n}, \mathrm{t})$ & ${ }^{6} \operatorname{Li}(\mathrm{n}, \mathrm{t})$ & ${ }^{7} \operatorname{Li}(\mathrm{n}, \mathrm{t})$ & ${ }^{7} \operatorname{Li}(\mathrm{n}, \mathrm{t})$ \\
& $\mathrm{D} 5$ & $\mathrm{D} 5$ & $\mathrm{D} 2$ & $\mathrm{D} 5$ & $\mathrm{D} 2$ & $\mathrm{D} 5$ \\
\hline JEFF 3.3 & 14.8 & 15.7 & 4.5 & 6.7 & 7.9 & 34.2 \\
\hline SCALE-6 & 6.3 & 18.6 & 5.4 & 9.7 & 9.0 & 24.1 \\
\hline ENDF/B-VIII* & 8.6 & 16.2 & 3.6 & 5.0 & 5.1 & 21.6 \\
\hline
\end{tabular}

* based on ${ }^{206,207,208} \mathrm{~Pb}$ covariances only

Table III. Total uncertainty in the calculated reaction rates for FNG HCLL benchmark based on JEFF3.3 and SCALE-5.0 covariance matrix evaluations (SUSD3D results based on the DORT neutron fluxes, $\mathrm{D} 1=3.5 \mathrm{~cm}, \mathrm{D} 7=28.5 \mathrm{~cm}, \mathrm{D} 8 \sim 33.55 \mathrm{~cm})$.

\begin{tabular}{|c|c|c|c|c|c|c|c|c|}
\hline \multirow{3}{*}{$\begin{array}{c}\text { HCLL } \\
\text { Covariance } \\
\text { data }\end{array}$} & \multicolumn{8}{|c|}{ Uncertainty (\%) } \\
\hline & ${ }^{115} \operatorname{In}\left(\mathrm{n}, \mathrm{n}^{\prime}\right)$ & ${ }^{27} \mathrm{Al}(\mathrm{n}, \alpha)$ & ${ }^{58} \mathrm{Ni}(\mathrm{n}, \mathrm{p})$ & ${ }^{93} \mathrm{Nb}(\mathrm{n}, 2 \mathrm{n})$ & ${ }^{6} \mathrm{Li}(\mathrm{n}, \mathrm{t})$ & ${ }^{6} \operatorname{Li}(n, t)$ & ${ }^{n a t} \mathrm{Li}(\mathrm{n}, \mathrm{t})$ & ${ }^{\text {nat }} \operatorname{Li}(n, t)$ \\
\hline & D8 & D8 & D8 & D8 & D1 & D7 & D1 & D7 \\
\hline ENDF/B-VI & 3.4 & 4.6 & 3.4 & 5.0 & 6.6 & 2.8 & 3.0 & 2.8 \\
\hline SCALE-6 & 2.8 & 7.1 & 4.9 & 7.2 & 2.2 & 1.4 & 1.2 & 1.2 \\
\hline
\end{tabular}




\section{CONCLUSIONS}

The computational scheme based on the SUSD3D sensitivity uncertainty code and TORT/GRTUNCL-3D deterministic transport codes was used in the pre-analysis of FNG-HCLL benchmark mock-up. The reaction rates and TPR were found to be rather strongly sensitive to the nuclear cross sections, in particular to the nuclear cross sections of $\mathrm{Pb}$ and $\mathrm{Fe}$. According to JEFF-3.3 covariance data the inelastic, $(\mathrm{n}, 2 \mathrm{n})$ and elastic cross sections on lead and iron isotopes were found to cause the highest uncertainty. The uncertainties due to cross section uncertainties were estimated to be around $15 \%(1 \sigma)$ for the In and Al reaction rates at the position around $50 \mathrm{~cm}$ from the $14 \mathrm{MeV}$ neutron source. Although the uncertainty in ${ }^{7} \mathrm{Li}$ tritium production is large, even above $30 \%$, the uncertainty in the tritium production for natural and $95 \%{ }^{6} \mathrm{Li}$ enriched detectors, which are planned to be used in the measurements, are limited to around 5-7\%.

The nuclear data uncertainties are considerably larger than the expected measurement uncertainties, which were typically of the order of around 5\% for the past FNG measurements. The above uncertainties are also considerably larger than those observed for example in the FNG HCLL benchmark measurements.

It is therefore anticipated that the proposed benchmark experiment is likely to contribute importantly to the validation and improvement of nuclear cross-section data and the corresponding covariance matrices used for fusion applications.

\section{ACKNOWLEDGMENTS}

This work has been carried out within the framework of the EUROfusion Consortium and has received funding from the Euratom research and training programme 2014-2018 and 2019-2020 under grant agreement No 633053. The views and opinions expressed herein do not necessarily reflect those of the European Commission.

\section{REFERENCES}

1. P. Batistoni, et al., Neutronics Experiment on a HCPB Breeder Blanket Mock-up, Fusion Engineering and Design, 82 (15), p.2095-2104, (2007)

2. I. Kodeli, Deterministic 3D Transport, Sensitivity and Uncertainty Analysis of TPR and Reaction Rate Measurements in HCPB Breeder Blanket Mock-up Benchmark, "Proc. Int. Conf. Nuclear Energy for New Europe 2006”, Portorož, Slovenia, Sept. 18-21, 2006

3. P. Batistoni, et al., "Neutronics experiments on HCPB and HCLL TBM mock-ups in preparation of nuclear measurements in ITER", International Symposium on Fusion Nuclear Technology (ISFNT 9), 11. - 16.10.2009, Dalian, China

4. P. Batistoni et al., "Design optimisation and measuring techniques for the neutronics experiment on a HCLL - TBM mock-up", Fus. Eng. Des. 84 (2009) 430-434

5. U. Fischer, P. Batistoni, A. Klix, I. Kodeli, D. Leichtle, R. L. Perel, "Neutronics R\&D efforts in support of the European breeder blanket development programme", Nucl. Fusion 49 (2009) 065009

6. I. Kodeli, et al., "Sensitivity and uncertainty analysis of the HCLL breeder blanket experiment in the frame of the EU fusion technology programme", Nuclear Eng. Design 241 (2011) 1243-1247.

7. D. Leichtle, U. Fischer, I. Kodeli, R. L. Perel, A. Klix, P. Batistoni, R. Villari, "Sensitivity and uncertainty analyses of the HCLL mock-up experiment", International Symposium on Fusion Nuclear Technology (ISFNT 9), 11. - 16.10.2009, Dalian, China

8. D. Flammini, et al., Pre-Analysis of the WCLL-Mock Up Neutronics Experiment at the Frascati Neutron Generator presented at the SOFT 2019 Conference, Budapest Sept. 22-27, 2019 
9. I. Kodeli, "Multidimensional Deterministic Nuclear Data Sensitivity and Uncertainty Code System, Method and Application", Nucl. Sci. Eng., 138 (2001), pp. 45-66.

10. I. Kodeli, "The SUSD3D Code for Cross-Section Sensitivity and Uncertainty Analysis - Recent Development", Trans. American Nuclear Society, Vol. 104, Hollywood, Florida, June 26-30, 2011.

11. R. L. Perel, Upgrading of the MCSEN sensitivity software to comply with the current standard of the MCNP-5 Monte Carlo code, Final report on Task 3.1 of the F4E Grant F4E-FPA-168.01, February 2014.

12. W. A. Rhoades, et al., DOORS 3.2, One-, Two-, Three-Dimensional Discrete Ordinates Neutron/Photon Transport Code System, CCC-650, Radiation Safety Information Computational Center, Oak Ridge National Laboratory (1998).

13. J. O. Johnson (Ed.), GRTUNCL3D, Code to Calculate Semi-Analytic First Collision Source and Uncollided Flux, ORNL, Oak Ridge (Jul 2004).

14. A. Koning et al., Status of the JEFF Nuclear Data Library, J. Korean Phys.Soc. 59,1057.

15. R.E. MacFarlane, TRANSX, Transport Cross Sections from MATXS Libraries, Vers. 2.15 (Nov. 94).

16. International Atomic Energy Agency: International Reactor Dosimetry File 2002 (IRDF-2002), Technical Reports Series No. 452, ISBN 92-0-105106-9, December 2006, “http://wwwpub.iaea.org/MTCD/publications/PDF/TRS452_web.pdf".

17. E. M. Zsolnay et al., Summary Description of the New International Reactor Dosimetry and Fusion File (IRDFF release 1.0), INDC(NDS)-0616, IAEA, Vienna, May 2012. http://wwwnds.iaea.org/IRDFF.

18. ZZ SCALE6.0/COVA-44G, 44-group cross section covariance matrix library extracted from SCALE6.0, NEA Data Bank, USCD-1236/02 (May 2009).

19. B. Kos, H. Sjostrand, I. A. Kodeli, JET contributors, Nuclear Data Uncertainty Propagation in Complex Fusion Geometries, this conference.

20. X-5 Monte Carlo Team, MCNP - A General Monte Carlo N-Particle Transport Code, Version 5, LAUR-03-1987, Los Alamos National Laboratory, Los Alamos, New Mexico, USA, (April 2003).

21. R. E. MacFarlane, D. W. Muir, The NJOY Nuclear Data Processing System, LA-12740-M, Los Alamos National Laboratory (1994). RSICC CODE PACKAGE PSR-368, 1999

22. I. Kodeli, ANGELO-LAMBDA, Covariance matrix interpolation and mathematical verification, NEADB Computer Code Collection, NEA-1798/02 (2008). 